\title{
Causes of Fasting-Evoked en Route Hypoglycemia in Diabetes (FEEHD): A Case Series Study
}

\author{
Saleh Aldasouqi ${ }^{1,2^{*}}$, Ved Gossain ${ }^{1,2}$, Matthew Hebdon ${ }^{1,2}$, Archana Reddy ${ }^{1,2}$, Jose Goldman ${ }^{1,2}$, \\ Sameer Ansar ${ }^{1,2}$, Bhavini Bhavsar ${ }^{1,2}$ \\ ${ }^{1}$ Department of Medicine, Michigan State University College of Human Medicine, East Lansing, Michigan, USA; ${ }^{2}$ Sparrow Diabetes \\ Center of Sparrow Hospital, Lansing, USA. \\ Email: *saleh.aldasouqi@ht.msu.edu
}

Received October $7^{\text {th }}, 2012$; revised November $15^{\text {th }}$, 2012; accepted November $28^{\text {th }}, 2012$

\begin{abstract}
Background: A recent study reported that some patients with diabetes who fast overnight for laboratory tests are at risk of developing hypoglycemia, which could occur while patients are in risky situations which could result in harm to patients or others. Due to limitation in study design of the aforementioned study, the causes of hypoglycemia could not be elucidated. Objective: To better understand fasting-evoked en route hypoglycemia in diabetes (FEEHD), a recently recognized overlooked safety problem in diabetes management. Methods and Patients: A recent study reported that some patients with diabetes who fast overnight for laboratory tests are at risk of developing hypoglycemia, which could occur while patients are in risky situations which could result in harm to patients or others. Due to limitation in study design of the aforementioned study, the causes of hypoglycemia could not be elucidated. We undertook this retrospective case series study, which enrolled 4 consecutive cases of fasting hypoglycemia in patients with diabetes who were on diverse antidiabetic medications. The study duration was June 1, 2010 to June 1, 2012. Results: The 4 cases (3 women, 1 man), with either type 1 or type 2 diabetes, revealed multiple defects in either the patients' knowledge and education regarding preparation for lab tests requiring fasting. The degree of hypoglycemia ranged from mild (65 mg/dl) to severe $(31 \mathrm{mg} / \mathrm{dl})$, and the events were either mildly symptomatic or asymptomatic. The possible causes of, and contributing factors to hypoglycemia are discussed, and recommendations for preventive measures are provided. Conclusion: Some patients with diabetes who fast for lab tests are at risk of hypoglycemia, and there seems to be an overall lack of proper education of patients with diabetes about this form of hypoglycemia. This overlooked problem can be prevented by proper education and preparation.
\end{abstract}

Keywords: Fasting; Hypoglycemia; Hypoglycemic Agents; Laboratory Tests; FEEHD

\section{Introduction}

Hypoglycemia is the major limiting factor towards achieving optimal control of hyperglycemia in patients with diabetes, which accounts for significant mortality and morbidity [1]. Recent literature [2-5] has uncovered an overlooked cause of iatrogenic hypoglycemia in patients with diabetes, which is overnight fasting for laboratory tests, which we will refer to as fasting-evoked en route hypoglycemia in diabetes (FEEHD). A fatal case report of FEEHD has been recently published [2,3].

In the first case report on FEEHD, retrieved upon an extensive literature search, Wiwanitkit described a fatal case of hypoglycemia in a patient who was taking sulfonylurea. However, this case report was briefly mentioned in 2 letters [2,3] and did not provide detailed description, other than that the patient was waiting in the

${ }^{*}$ Corresponding author. laboratory for a lab test, while fasting, when she had a sudden cardiac arrest, and could not be resuscitated, and that the cause of death was severe hypoglycemia, with blood glucose of 0 (zero) $\mathrm{mg} / \mathrm{dl}$.

In the only study published to address FEEHD, Aldasouqi et al. [4] reported that some of their patients with both type 1 (T1DM) and type 2 diabetes (T2DM) developed hypoglycemia when they fasted overnight for laboratory tests. In that study, the authors reported that the hypoglycemia observed was variable in symptoms and in severity, with glucose as low as $31 \mathrm{mg} / \mathrm{dl}$ in one incident. The impetus for the study [4] was that such patients would be at risk of harm (to themselves or to others) should their hypoglycemia rapidly progress while driving en route, to and from the laboratory facilities.

However, due to limitations in the design of the aforementioned retrospective pilot study (related to reliance on patients' remote recollection), the authors could not 
evaluate the circumstances or etiologies contributing to the reported hypoglycemic incidents. The authors reported new preventive measures that they implemented in their practice [4]. In a follow up study recently presented at a national endocrinology annual meeting in the USA [5], the same authors reported significant reduction of FEEHD, which they attributed to increased awareness of the problem and the implementation of the prevention program.

Becoming aware of the aforementioned new observation, and in a different clinical setting (the Sparrow Diabetes Center (SDC) of Sparrow Hospital, Lansing, Michigan, affiliated with Michigan State University (MSU), East Lansing, Michigan, USA), we developed similar preventive measures. This preventive program was implemented after undertaking an internal quality improvement audit, which confirmed the occurrence of FEEHD in patients attending the SDC and MSU endocrinology clinics (unpublished data). At the same time, we began to carefully evaluate any subsequent incidental cases of FEEHD. Given this advantage of capturing such events upon occurrence, we were able to accurately document the cases with recording of all possible related circumstances that could contribute to this form of hypoglycemia. Furthermore, we were also able to provide immediate evaluation and management of such case upon occurrence.

In this case series, we describe 4 cases of FEEHD that were captured recently, where it was feasible to obtain detailed case description. This retrospective case series study was not designed to evaluate the prevalence or frequency of FEEHD. The study was approved by the Institutional Review Boards of MSU and Sparrow Hospital. The study duration was June 1, 2010 to June 1, 2012.

\section{Case Presentations}

In this report, we describe the demographical, clinical and laboratory findings of 4 consecutive cases, recently encountered at our diabetes and endocrine clinics. In this case study, we were able to evaluate the cases more precisely, given recent event occurrence (3 cases) and/or patients' vivid recollection of the hypoglycemic incidents (1 case).

The 4 cases of hypoglycemia reported herein were carefully analyzed, in regards to the circumstances surrounding the hypoglycemic episodes that were confirmed by the laboratory. The patients were able to give details about the hypoglycemic events. The cases are described in in Tables 1 and 2. All the lab tests were obtained in the morning after overnight fasting, and the original order was for lipid profile, or other lab orders including chemistry. The following is further analysis of the cases, in respect to the hypoglycemic episodes:

\section{Case 1:}

This patient had new onset T1DM. She was obviously insulin sensitive (thin, active), with frequent hypoglycemic episodes on the regimen, since her discharge, about 3 weeks prior to her office visit. She was referred to our diabetes clinic after a recent hospitalization, and was on Glargine 20 units at night, and Aspart pre-meal 3 - 4 units. In view of the hypoglycemic episodes, her Glargine and Aspart doses were empirically reduced to 12 and 2, respectively, with adjustment of pre-meal insulin according to meals and correction requirements, and she was instructed about detection and treatment of hypoglycemia. She also received full diabetes education during the consultation office visit, but this did not include instructions about fasting labs, although a fasting lab order was issued and given to her upon discharge from the clinic. The 12-hours overnight fasting was for the purpose of a lipid test.

She went for the lab a week later. She took the night dose of Glargine, but she did not take the short-acting insulin since she knew she should not take it without eating on the morning of the test... On the day of the test, her fasting blood sugar (FBS) was $78 \mathrm{mg} / \mathrm{dl}$ (6:30 AM), before she left to the lab and she was feeling well, and thus she thought she would be safe to drive to the lab. Later, and while she was in the lab, she had hypoglycemic symptoms. Soon afterwards, she managed to drive to a nearby restaurant and had a breakfast, after which she felt better.

Case 2:

This woman with T2DM was referred for a thyroid problem. During her office visit, the hypoglycemic result was noted on her forwarded lab tests (from 2 weeks prior). This was further evaluated, and it was noted that she was on Glyburide, which she usually took in the evening, and also did so before the lab test. The patient stated she was not told previously to take the Glyburide in the morning.

Case 3:

Despite a 33-year history of T2DM, this retired, educated man stated that he had never been educated on the concept of skipping insulin in the morning, when fasting overnight for lab tests. This represents a clear gap in diabetes education, although he reported attending classes over the years, and considered himself well-educated in diabetes self-management. Obviously, he was completely asymptomatic despite the severe degree of his hypoglycemia (31 mg/dl). From prior similar hypoglycemic lab reports (that he and his health providers had been aware of) over the years, he has learned to carry snacks in the car, so he will eat immediately after the lab draws.

\section{Case 4:}

This patient with T2DM of long-duration was not asked to fast for the morning blood tests, but she volun- 
Table 1. Demographical and clinical data of patients who fasted overnight in preparation for laboratory tests.

\begin{tabular}{|c|c|c|c|c|}
\hline Case \# & 1 & 2 & 3 & 4 \\
\hline Age (years) & 35 & 73 & 68 & 65 \\
\hline Type of diabetes & 1 (on insulin) & 2 (OHAs, no insulin) & 2 (Insulin requiring) & 2 (Insulin requiring) \\
\hline Duration of diabetes & New onset & $>10$ years & 33 years & 31 years \\
\hline Glycohemoglobin A1c & $6.7 \%$ & $6.4 \%$ & $7.6 \%$ & $10.3 \%$ \\
\hline $\begin{array}{l}\text { Impaired kidney or liver } \\
\text { function, or contributing } \\
\text { clinical disorders }\end{array}$ & No & No & No & No \\
\hline Anti-diabetic medications & $\begin{array}{c}\text { Glargine, Insulin } \\
\text { Aspart }\end{array}$ & Glyburide, metfromin, & $\begin{array}{l}\text { Detemir, humilin R } \\
\text { metformin, }\end{array}$ & $\begin{array}{c}\text { Glargine, insulin lispro, } \\
\text { metformin }\end{array}$ \\
\hline Other medications & None & $\begin{array}{c}\text { Simvastatin, chlorthallidone, } \\
\text { losartan, montelukast, } \\
\text { levothyroxine, } \\
\text { fluticasone-salmeterol, } \\
\text { zolpidem, albuterol, } \\
\text { clonidine, amlodipine, prazosin }\end{array}$ & $\begin{array}{l}\text { Fosinopril, metformin, } \\
\text { hydrochlorothiazide, } \\
\text { diltiazem, ezetimibe }\end{array}$ & $\begin{array}{l}\text { Simvastatin, aspirin, } \\
\text { ezetimibe, enalapril, } \\
\text { hydrocholorothiazide }\end{array}$ \\
\hline
\end{tabular}

Table 2. Description of the hypoglycemic events in patients who fasted overnight in preparation for laboratory tests.

\begin{tabular}{|c|c|c|c|c|}
\hline Case \# & 1 & 2 & 3 & 4 \\
\hline Glucose (mg/dl) & 61 & 65 & 31 & 48 \\
\hline $\begin{array}{l}\text { Hypoglycemic } \\
\text { symptoms }\end{array}$ & $\begin{array}{l}\text { Extreme hunger, } \\
\text { anxiety, shakes, } \\
\text { headache }\end{array}$ & $\begin{array}{l}\text { No symptoms (mild } \\
\text { hypoglycemia, long } \\
\text { duration of diabetes) }\end{array}$ & $\begin{array}{l}\text { No symptoms (long duration } \\
\text { of diabetes with obvious } \\
\text { hypoglycemia unawareness) }\end{array}$ & $\begin{array}{c}\text { No symptoms (long } \\
\text { duration of diabetes with } \\
\text { obvious hypoglycemia } \\
\text { unawareness) }\end{array}$ \\
\hline Consciousness & Maintained & Maintained & Maintained & Maintained \\
\hline $\begin{array}{l}\text { Factors contributing to } \\
\text { hypoglycemia during } \\
\text { fasting beyond } \\
\text { breakfast time }\end{array}$ & $\begin{array}{l}\text { Insulin sensitivity, } \\
\text { new onset diabetes } \\
\text { with inadequate } \\
\text { education }\end{array}$ & $\begin{array}{l}\text { Taking long-acting } \\
\text { sulfonylurea, dosing of } \\
\text { sulfonylurea in the PM }\end{array}$ & $\begin{array}{l}\text { Inadequate education about } \\
\text { holding insulin when fasting, } \\
\text { which patient said he had } \\
\text { always done when going for } \\
\text { fasting labs }\end{array}$ & $\begin{array}{l}\text { Interestingly, the patient } \\
\text { was not asked to fast, but } \\
\text { volunteered to fast, given } \\
\text { prior lab experiences }\end{array}$ \\
\hline $\begin{array}{c}\text { Education about } \\
\text { fasting for lab tests }\end{array}$ & $\begin{array}{l}\text { Not provided before } \\
\text { the lab test, but } \\
\text { afterwards: No more } \\
\text { fasting labs (lipid } \\
\text { profile normal), and if } \\
\text { needed, to order } \\
\text { non-fasting, non-HDL } \\
\text { lipid formula; to } \\
\text { monitor glucose on } \\
\text { the day of test }\end{array}$ & $\begin{array}{l}\text { Not provided before the lab } \\
\text { test, but afterwards: change } \\
\text { sulfonylurea dosing to } \\
\text { morning, and to switch to a } \\
\text { non-long-acting sulfonylurea; } \\
\text { to monitor glucose before } \\
\text { and during the lab test }\end{array}$ & $\begin{array}{l}\text { Not provided before the lab } \\
\text { test, but afterwards: To avoid } \\
\text { taking insulin when fasting for } \\
\text { labs; to minimize ordering } \\
\text { fasting labs; to monitor } \\
\text { glucose before and during the } \\
\text { lab test }\end{array}$ & $\begin{array}{l}\text { Not provided before the } \\
\text { lab test, but afterwards: } \\
\text { She was instructed never } \\
\text { to fast unless ordered; and } \\
\text { if so, to contact the } \\
\text { diabetes center for } \\
\text { medications adjustment }\end{array}$ \\
\hline
\end{tabular}

teered to do so based on prior experiences with lab testsalthough the labs ordered this time did not require fasting. She was taking Metformin 1000 mg twice daily, Glargine 64 units in the AM and 54 units in the PM, and Lispro 10-14 units before meals. She took the full dose of Glargine the night before the test, as well as the PM doses of Metformin and Lispro.

\section{Cases 1 - 4:}

Upon recognition of the hypoglycemic events, each patient was educated and instructed individually regarding proper preparation for fasting labs when deemed necessary. Instructions were based on recommendations by 
Aldasouqi et al. [4]. Proposed recommendations are summarized below:

1) If possible, patients should avoid fasting for laboratory tests, such as testing non-fasting lipids (such as non-HDL cholesterol), rather than fasting lipid profile.

2) If fasting is unavoidable:

a. Patients should test sugars more often upon awakening, and during the trip to the lab, if needed.

b. Patients to cancel the lab test if the sugar drops, or if too high to require treatment especially in patients with T1DM.

c. Patients to carry sugar tablets with them in case of hypoglycemia.

d. Patients should not drive while fasting, but to bring a companion who can manage hypoglycemia.

e. To avoid all types of insulin in the AM while fasting: This may be a problem in T1DM taking AM basal insulin, due to concerns about hyperglycemic crisis. Individualization is advisable. Short-acting insulin certainly should be skipped.

f. Patients should cut the night dose of basal insulin by $10 \%-30 \%$. This should also be individualized.

g. Similarly insulin pump basal rate should be reduced by $10 \%-30 \%$ during the fasting period. This should also be individualized.

PM doses of Sulfonylureas should be held the night before, and certainly AM doses should be deferred on the morning of the lab test until fasting is completed.

\section{Discussion}

This study has uniquely captured a series of 4 cases that encompass a diverse range of demographic and clinical circumstances which could cover most possible factors potentially related to hypoglycemia in diabetes: Age, gender, medications, co-morbidities, hypoglycemia unawareness, and other factors related to diabetes education (or lack thereof) by patients or healthcare professionals. The common denominator is the overnight fasting. While hypoglycemia of diabetes is a well-described topic in the medical literature, this form of hypoglycemia, FEEHD, that we have recently recognized $[4,5]$ has not been addressed prior. While it could be argued that patients with diabetes may ultimately have hypoglycemia whether or not they fast for lab tests, the main concern about the potential seriousness of FEEHD is that patients may be at higher risks than other forms of hypoglycemia, as explained below.

The prevalence or seriousness of FEEHD is not known. Despite the paucity of relevant literature, a fatal case has been documented in the literature, as recently reported by Wiwanitkit [2,3]. However, more literature about diabetes-associated hypoglycemia (in general) is emerging, which is concerning. A recent national survey of 2530 participants [6] has indicated that many patients with diabetes remained uneducated about the risks of hypoglycemia. The survey highlighted why hypoglycemia may be a more serious health hazard than previously reported, as patients indicated they often had hypoglycemia while working or driving. We believe that FEEHD is a strikingly overlooked problem, overall, and that patients with diabetes are not properly educated about it. Patients with diabetes may undergo fasting for various purposes, and guidelines are available in the literature for such purposes, such as overnight fasting for surgical and diagnostic procedures [7-9], and fasting for the purpose of religious observance $[10,11]$.

Such guidelines addressing FEEHD are lacking in the medical literature on diabetes and diabetes education. It is prudent to include this education piece in discussions with patients with diabetes, by their clinicians, nurses, educators, and pharmacists. Stemming from lack of awareness about FEEHD, it is customary for clinicians to "routinely" order lipid profiles with an average of 8 - 12 hours of fasting, without giving patients instructions regarding glucose monitoring or adjustment of anti-diabetic medications, in anticipation of fasting.

The unique situation about FEEHD as compared to other scenarios of hypoglycemia is that patients may develop serious hypoglycemia while en route to lab facilities, either going into the facility, or rushing out to have a meal after phlebotomy. To practice adherence, the patients may feel obliged to continue fasting, even if they feel symptoms - since they were given a (strict) order for fasting. Case 4 in this series illustrates such extreme adherence to fasting, which goes beyond labs traditionally requiring fasting (e.g., lipid profiles) to labs not requiring fasting (chemistry), and even without being asked to fast. If patients have hypoglycemia unawareness, or if the phlebotomy is delayed further, due to patients' or facility's reasons, the risk of hypoglycemia will be higher and the resulting harm more significant. It can be conceived that the risk of hypoglycemia when fasting for lab tests could be worse than fasting for surgical or diagnostic procedures.

The reason why we believe that FEEHD is potentially worse than the other known fasting circumstances (e.g. surgery) is that patients are presumably educated on proper preparation, and would be presumably well-monitored during surgery. In contrast, patients who develop FEEHD could be in hazardous environmental circumstances. This scenario is potentially serious. If hypoglycemia is severe while patients are driving en-route to and from lab facilities, this can result in harm to the patient or others if it causes traffic accidents [6,7].

People without diabetes can tolerate prolonged periods of fasting without hypoglycemia [10-16], provided they have normal physiological homeostasis. During fasting, 
the body goes into 3 metabolic phases [15]: post-absorbtive, gluconeogenic and protein conserving. For people to tolerate fasting, beyond the usual 8 hours of overnight sleep, the body's ability to withstand prolonged fasting requires basic physiological defensive mechanisms (barriers) that should protect the body against hypoglycemia, which include gradual reduction in insulin levels towards the nadir, and variable increases in glucagon, epinephrine, cortisol and growth hormone [15-17]. These protective counter-regulatory mechanisms can be impaired in significant numbers of patients with diabetes, especially in patients with advanced or complicated diabetes [15,17]. With continued insulin availability (native, via insulin secretagouges or exogenous), the continued physiologic insulin action will prevent optimal glucose release from the liver. All of this coupled with waning hepatic glycolgen stores upon prolonged fasting (12 hours or more), the risk of hypoglycemia is heightened in certain patients with diabetes upon fasting.

Since the issue of FEEHD has not been addressed in the literature, we evaluated in this case series the circumstances that could (hypothetically) contribute to hypoglycemia in our patients, which accentuated the effect of skipping their breakfast. It has been noted that the development of hypoglycemia is almost inevitable in any patient with diabetes who is on treatment, at one point. In this regard, Fowler used an interesting phrase to underscore this observation [17], by stating that "Hypoglycemia in many ways is the Achilles heel of diabetes treatment". Any anti-diabetic medication can potentially cause hypoglycemia, but insulin and secretagouges, certainly are the usual culprits in clinical practice.

In case 1 , we hypothesize that lingering levels of basal insulin in the face of waning hepatic glucose stores upon fasting could have caused hypoglycemia. Obviously, while the bed-time dosing of Glargine resulted in reasonable FBS around the usual time of her breakfast, the continued fasting thereafter precipitated the hypoglycemic episode; her usual breakfast time is 4:30 AM. However, she thought that her sugar was fine before she left home $(78 \mathrm{mg} / \mathrm{dl})$. It is conceivable thus that additional fasting beyond her usual breakfast time, of (about 3.5 hours) precipitated her hypoglycemic episode.

Basal insulins theoretically are not supposed to have peak effects, and are supposed to provide stable low levels of insulin, and hence the concept of being a (basal insulin), and thus the projected lowered incidence of hypoglycemia. This was tested clinically in 15 patients with T1DM taking a basal insulin, during an 18 hours period of fasting [18], with only 2 minor hypoglycemic events. However, the aforementioned study cohort was carefully selected-unlike in real life situations (advanced age, variable degrees of glycemic control, insulin dosing, level of education, presence of co-morbidities, etc.). Therefore, it is hard to extrapolate the conclusions from this small study on basal insulins to the general population.

In case 2, we hypothesize that Glyburide, which is a long-acting sulfonylurea (SU), is expected to have lingering, but significant effects through the next day, when given at night. We thus hypothesized that the secretagogue effect of Glyburide continued to pour more insulin into the blood stream in the face of waning glucose release from the liver upon fasting.

In case 3, the cause of hypoglycemia is the effects of both basal and rapid action insulin taken in the morning of the test, while fasting. The educational gap, as discussed earlier, is the main culprit as the etiology of his hypoglycemia. The patient has obvious hypoglycemia unawareness (asymptomatic despite glucose of $31 \mathrm{mg} / \mathrm{dl}$ ).

And finally, in case 4, it is hypothesized that the fasting hypoglycemia was caused by lingering effects of Glargine, taken the night before. Intriguely, this case illustrates the religious adherence by patients to the (doctor's order) of fasting for lab tests. The patient was not required to fast, and the lab order did not include lipid profile which is the usual test for which clinicians insist on the fasting prerequisite. But the patient volunteered to fast on her own. We even have anecdotally observed few other patients coming fasting to their diabetic follow up visit, in case the doctor would need a fasting test!

Given the aforementioned diverse circumstances and patients' characteristics, we believe that this case series encompasses the various major circumstances that could contribute to FEEHD, These cases reveal multiple defects in diabetes education, and at many levels, ranging from patients to some members of the healthcare personnel, including diabetes educators, nurses and clinicians. While this case series describes circumstances noticed in our patient population, we believe these circumstances may apply universally to patients with diabetes; however, we can not ascertain that and further research by other investigators is warranted.

Finally, we believe that if patients with diabetes who are requested to fast for lab tests are educated adequately and prepared properly, the risk of FEEHD can be reduced or eliminated.

\section{Conclusion}

In conclusion, this case series suggests multiple educational defects in diabetes management that may result in FEEHD, an overlooked and serious safety problem in patients with T1DM and T2DM, and patients should be better educated about this piece of diabetes education.

\section{Authors' Contributions}

Author SA designed the study, searched the literature, researched data, contributed to discussion, and wrote/ 
reviewed/edited the manuscript. Co-Authors VG, MH, $\mathrm{AR}, \mathrm{JG}, \mathrm{SA}$ and $\mathrm{BB}$ contributed to study planning and conduction and to research discussions.

\section{Acknowledgements}

Parts of this case series were presented as posters at the 2012 annual meeting of the American Association of Clinical Endocrinologists (AACE), in Philadelphia, PA, May 23-27, 2012.

The authors would like to thank the following experts for their thoughtful inputs and suggestions: Mary Austin, MA, RD, CDE, FAADE (Past President of the American Association of Diabetes Educators (AADE), Shelby Township, Michigan; Martin Grajower, MD, FACE, FACP (Division of Endocrinology, Department of Medicine, Albert Einstein College of Medicine, Bronx, NY); and Anne Peters, MD, Professor of Medicine and Director of the University of Southern California Diabetes Program, Los Angeles, California.

The authors would like also to thank Jinie Shirey at the Department of Medicine, MSU, for assistance secretarial assistance, and the following senior librarians, for their efforts in literature search: Laura Smith, Mike Simmons, and Steve Kalis (Medical Library of Sparrow Hospital, Lansing, Michigan); and Faith Chrutcheon (Medical Library of Saint Francis Medical Center, Cape Girardeau, Missouri). Finally, the authors would also like to thank Kim Mathew (Director of the Chemistry Section of the Saint Francis Medical Center Laboratory, Cape Girardeau, Missouri) for her assistance with laboratory data retrieval.

\section{Financial Support and Disclosures}

This case series was not sponsored or funded by any commercial entity. Co-author SA received research grant from Amylin for an observational 2-year multi-center study on exenatide (2008-2010) and received honoraria from Takeda, Novo Nordisk and Merck. Co-author VG received research grants and support from Eli Lilly and UAW-GM for unrelated research projects. No other potential conflicts of interest relevant to this article were reported.

\section{REFERENCES}

[1] The American Diabetes Association Workgroup on Hypoglycemia, “Defining and Reporting Hypoglycemia in Diabetes: A Report from the American Diabetes Association Workgroup on Hypoglycemia,” Diabetes Care, Vol. 28, No. 5, 2005, pp. 1245-1249. doi:10.2337/diacare.28.5.1245

[2] V. Wiwanitkit, "Case of Sudden Death in Venipuncture Clinic,” Phlebotomy, Vol. 19, No. 4, 2004, p. 193. doi:10.1258/0268355042554993

[3] V. Wiwanitkit, "A Problematic Case Due to Blood Collection for Fasting Plasma Glucose Assessment,” Shiraz
E Medical Journal, Vol. 7, No. 3, 2006, p. 1.

[4] S. Aldasouqi, A. Sheikh, P. Klosterman, S. Kniested, L. Schubert, R. Danker and M. Austin, "Hypoglycemia in Patients with Diabetes on Antidiabetic Medications Who Fast for Laboratory Tests,” Diabetes Care, Vol. 34, No. 5, 2011, p. e52. doi:10.2337/dc10-2402

[5] S. Aldasouqi, A. Sheikh, P. Klosterman, S. Kniested, L. Schubert, R. Danker, M. Austin and M. Grajower, "Hypoglycemia While Fasting for Morning Blood Tests: A Follow up of the Cape Girardeau Hypoglycemia en Route Prevention Program,” Proceedings of the Annual Meeting of the American Association of Clinical Endocrinologists, Philadelphia, 23-27 May 2012.

[6] B. Bouton, "Patients with Diabetes Lack Knowledge about Hypoglycemia,” Medscape Website http://www.medscape.com/viewarticle/740881

[7] American Diabetes Association, "Standards of Care in Diabetes - 2011: Hypoglycemia,” Diabetes Care, Vol. 34, Suppl. 1, 2011, pp. S25-S26.

[8] American Association of Diabetes Educators, "The Art and Science of Diabetes Self-Management Education: A Desk Reference for Healthcare Professionals,” In: C. Mensing, Ed., American Association of Diabetes Educators, Chicago, 2006.

[9] S. Degogo-Jack and K. Alberti, "Management of Diabetes Mellitus in Surgical Patients,” Diabetes Spectrum, Vol. 15, No. 1, 2002, pp. 44-48. doi:10.2337/diaspect.15.1.44

[10] M. Al-Arouj, R. Bouguerra, J. Buse, et al., "Recommendations for Management of Diabetes during Ramadan," Diabetes Care, Vol. 28, No. 9, 2005, pp. 2305-2311. doi:10.2337/diacare.28.9.2305

[11] M. M. Grajower, "Management of Diabetes Mellitus on Yom Kippur and Other Jewish Fast Days,” Endocrine Practice, Vol. 14, No. 3, 2008, pp. 305-311.

[12] M. Korbonits, D. Blaine, M. Elia and J. Powel-Tuck, "Refeeding David Blaine-Studies after a 44 Day-Fast," New England Journal of Medicine, Vol. 353, No. 21, 2005, pp. 2306-2307. doi:10.1056/NEJM200511243532124

[13] J. Reiter, I. D. Wexler, N. Shehadeh, A. Tzurt and D. Zangen, “Type 1 Diabetes and Prolonged Fasting," Diabetic Medicine, Vol. 24, No. 4, 2007, pp. 436-439. doi:10.1111/j.1464-5491.2007.02098.x

[14] M. Grajower, "24-Hour Fasting with Diabetes: Guide to Physicians Advising Patients on Medication Adjustments Prior to Religious Observances (or Outpatient Surgical Procedures)," Diabetes/Metabolism Research and Reviews, Vol. 27, No. 5, 2011, pp. 413-418. doi:10.1002/dmrr.1169

[15] M. Al-Arouj, S. Assaad-Khalil, J. Buse, et al., "Recommendations for Management of Diabetes during Ramadan: Update 2010,” Diabetes Care, Vol. 33, No. 8, 2010, pp. 1895-1902. doi:10.2337/dc10-0896

[16] J. Hoeks, N. A. van Herpen, M. Mensink, E. MoonenKornips, D. van Beurden, M. K. Hesselink and P. Schrauwen, "Prolonged Fasting Identifies Skeletal Muscle Mitochondrial Dysfunction as Consequence Rather than Cause of Human Insulin Resistance,” Diabetes, Vol. 59, No. 9, 2010, pp. 2117-2125. doi:10.2337/db10-0519 
[17] M. Fowler, "The Diabetes Treatment Trap: Hypoglycemia,” Clinical Diabetes, Vol. 29, No. 1, 2011, pp. 36-39. doi:10.2337/diaclin.29.1.36

[18] G. Mucha, S. Merkel, W. Thomas and J. Bantle, "Fasting and Insulin Glargine in Individuals with Type 1 Diabetes,” Diabetes Care, Vol. 27, No. 5, 2004, pp. 1209-1210. doi:10.2337/diacare.27.5.1209

\section{List of Abbreviations}

FEEHD: fasting-evoked en route hypoglycemia in diabetes.

T1DM: Type 1 diabetes mellitus.

T2DM: Type 2 diabetes mellitus. 